Progress in Flight Physics 5 (2013) 309-326

DOI: $10.1051 /$ eucass/201305309

C Owned by the authors, published by EDP Sciences, 2013

\title{
LARGE-EDDY SIMULATION OF HIGH MACH NUMBER FILM COOLING WITH SHOCK-WAVE INTERACTION
}

\section{Konopka, M. Meinke, and W. Schröder}

Institute of Aerodynamics, RWTH Aachen University

5a Wüllnerstr., Aachen 52062, Germany

\begin{abstract}
The impact of shock waves on supersonic cooling films is studied using large-eddy simulations (LES). A laminar cooling film is injected through a slot at a Mach number $\mathrm{Ma}_{i}=1.8$ into a fully turbulent boundary layer at a freestream Mach number $\mathrm{Ma}_{\infty}=2.44$. The cooling film is disturbed by oblique shock waves at deflection angles of $5^{\circ}$ and $8^{\circ}$ at two downstream positions of the slot. At shock impingement close to the slot, i. e., within the potential-core region, at a flow deflection of $5^{\circ}$, a cooling effectiveness decrease of $33 \%$ occurs downstream of the separation bubble compared to a configuration without shock impingement. If the same shock impinges further downstream upon the boundary-layer region, the decrease is only $17 \%$. The stronger 8 degree shock wave at the further downstream impingement position leads to a maximum decrease of $33 \%$.
\end{abstract}

\section{INTRODUCTION}

In supersonic combustion ramjets (scramjets), shock waves are present in the isolator and combustion chamber. These shock waves interact with cooling films if supersonic film cooling is used to protect the engine's interior surfaces from the intense aerodynamic heating and hot combustion products. This film cooling problem, i. e., the interaction of shock waves with a supersonic cooling film injected through a slot, was investigated experimentally for turbulent flows [1-3] to assess the impact on the heat transfer and cooling effectiveness. More recent experimental studies performed by Kanda et al. [4, 5] on film cooling with shock wave interaction found that shock waves have only a little effect on the cooling effectiveness. Kanda and Ono and Kanda et al. showed that the cooling effectiveness is mainly reduced by the increased wall-recovery temperature which is caused by the reduced local Mach number downstream of the shock wave. However, Peng and Jiang [6] found in their computational study using 


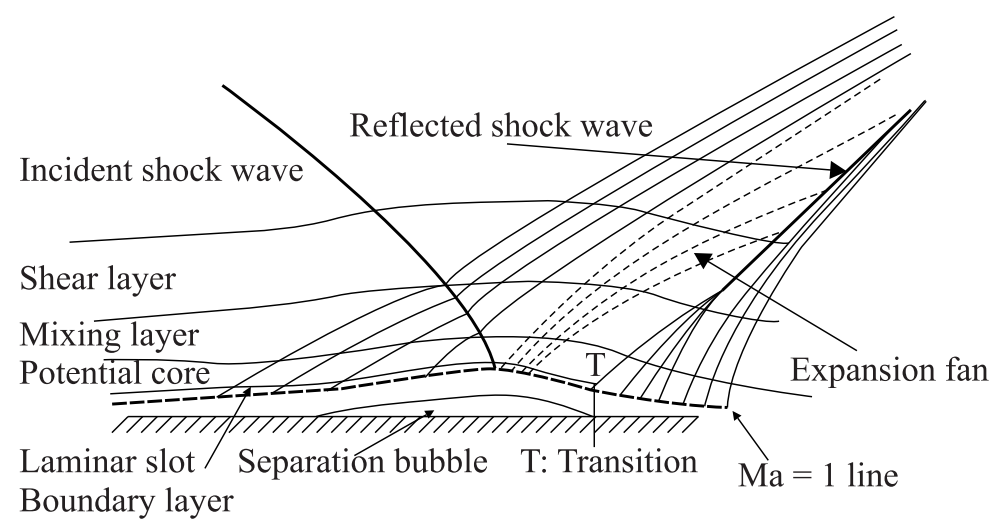

Figure 1 Main flow features of shock-wave impingement upon the potential-core region (case II)

the Reynolds-averaged Navier-Stokes (RANS) equations with the Menter SST (Shear Stress Transport) [7] turbulence model that the mole fraction of the injected gas decreased at the impingement position of the shock waves. This indicates that increased mixing due to the excited turbulence levels in the cooling flow occurred additionally to the effect of the reduction of the local Mach number. Therefore, the following study focuses on whether the reduction of cooling effectiveness by shock waves is only a function of the local Mach number or if increased turbulence plays a role. Five cooling configurations at the same injection condition are investigated. A zero pressure gradient configuration (case I) is compared to experiments of Juhany and Hunt [8]. Two configurations where a shock wave is generated by a flow deflection angle of $5^{\circ}$ at varying shockimpingement positions are analyzed. The first impingement position is located within the potential-core region $[9,10]$, where the injected cooling flow is laminar (case II). The second position is located further downstream in the boundarylayer region $[8,9]$ (case III), where the cooling flow mixes with the freestream and boundary-layer-like velocity profiles occur. Additionally, a stronger shock wave generated by an $8^{\circ}$ flow deflection impinges at the same downstream position within the boundary-layer region upon the cooling flow (case IV).

The principal flow features of shock-cooling film interactions within the potential-core region (case I) are sketched in Fig. 1. The potential-core region, which originates at the slot, is encompassed by the laminar slot boundary layer and the mixing layer where the freestream mixes with the cooling flow. On top of the mixing layer, there is the shear layer which emanates from the lip and is fed by the turbulent boundary layer. The incident oblique shock wave crosses these layers and causes the laminar separation bubble. Slightly upstream of the separation bubble compression waves decelerate the flow, at the crest of the bub- 
ble an expansion fan emerges, and then the flow reattaches creating compression waves which unify and form a shock wave. At the end of the separation bubble, the laminar slot boundary layer undergoes transition.

The paper is organized as follows. First, the numerical method will be presented, subsequently, details of the boundary conditions and the computational mesh will be given. Next, the flow configuration is explained and the results section follows. In the results section, the zero pressure gradient film cooling configuration is validated by experiments from Juhany and Hunt [8], then, shock-cooling-film interactions are investigated in terms of cooling effectiveness, instantaneous flow properties, and turbulence statistics. Finally, some conclusions are drawn.

\section{NUMERICAL METHOD}

In the past, RANS equations using among other approaches $k-\varepsilon$ turbulence models were used [11-13] to model film cooling problems with varying success, i. e., depending on the variant of the model, there was quite a discrepancy in the wall temperature distributions. This is caused in part by the modeling of the mixing layer between the cooling flow and the freestream, where no satisfying model to account for density gradients exist [14]. Therefore, in this study, high fidelity turbulence modeling is applied, i. e., LES are performed.

The Navier-Stokes equations are discretized at second-order accuracy using a modified mixed-centered upwind advective upstream splitting method (AUSM) [15] for the Euler terms. The discretization of the non-Euler terms is done using a centered approximation at second-order accuracy. The temporal integration is done by a second-order five-stage low-storage Runge-Kutta method. The nonresolved subgrid scales are implicitly modeled using the multiple integrated LES ansatz [16]. The viscosity is evaluated by a power law $\mu / \mu_{0}=\left(T / T_{0}\right)^{0.72}$ where $T_{0}$ denotes the stagnation temperature. A detailed summary of the flow solver used in this study is given by Meinke et al. [17]. The accuracy of its solutions in fully turbulent flows is discussed in [18-20]. The solution algorithm has also shown convincing results in supersonic flows involving shock-boundary layer interactions [21].

\section{BOUNDARY CONDITIONS AND COMPUTATIONAL MESH}

The prescription of realistic inflow variables for compressible turbulent boundary layers is a challenge in LES since at every time step a different instantaneous inflow distribution is required. This problem can be solved by computing the 


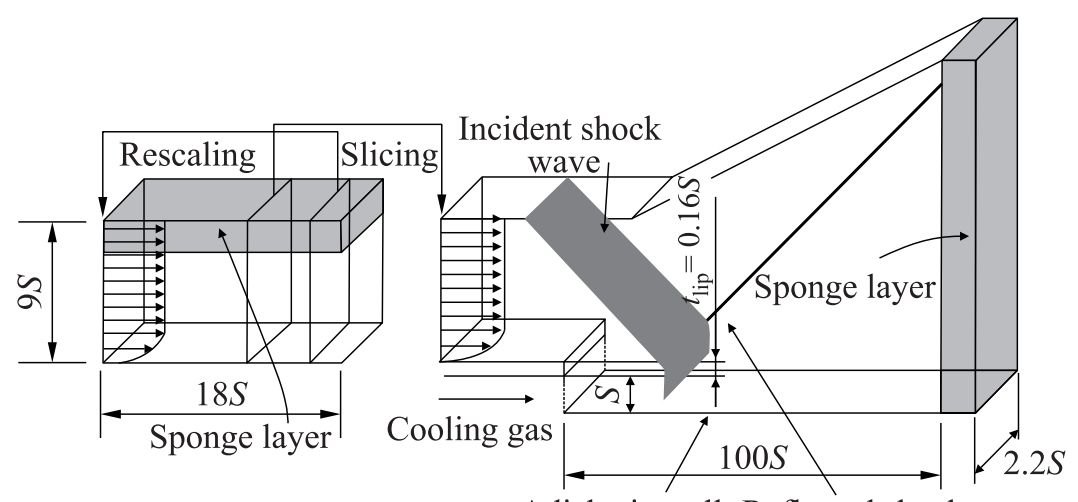

Adiabatic wall Reflected shock wave

(a)

(b)

Figure 2 Sketch of the boundary conditions and the turbulent inflow data generation method: (a) boundary-layer simulation; and (b) film-cooling simulation

compressible turbulent boundary layer from the leading edge of a flat plate. To avoid this computationally costly approach, an independent boundary layer simulation is performed using the rescaling method proposed by El-Askary et al. [21], which is based on Lund et al.'s [22] approach considering compressibility. In the boundary-layer domain, which is depicted in Fig. 2, the inflow distribution is generated by rescaling the flow variables obtained from a plane within the domain so that a constant boundary layer thickness at the inflow is achieved. A slice of the flow field is then extracted at every time step and injected into the main film cooling domain.

At the lower wall of the film-cooling simulation domain adiabatic no-slip boundary conditions are imposed, at the exit all variables are extrapolated. To avoid any spurious oscillations, a sponge layer is used at the exit and at the top of the boundary-layer domain where the flow variables are driven to the desired target variables [21]. At the slot, a laminar supersonic cooling flow is prescribed. In the spanwise direction, periodic boundary conditions are used. The desired shock-wave strength and angle are generated at the upper boundary by setting flow conditions which satisfy the Rankine-Hugoniot relations.

The body-fitted computational mesh consists of 17.1 million grid points with an equidistant spacing in the streamwise and spanwise directions. The resolution at the wall in inner coordinates is $\Delta x^{+}=20, \Delta y^{+}=0.5, \Delta z^{+}=10$ in the streamwise, wall-normal, and spanwise directions. A grid study for this cooling configuration was performed by Konopka et al. [23] where the current resolution was found to be adequate. Due to the spanwise domain size of $z / S=2.2$, the computations do not resolve some large-scale vortices. A similar cooling configuration with a lower injection Mach number of $\mathrm{Ma}_{i}=1.2$ was investigated 
by Konopka et al. [24] and it was found that the spanwise domain size of the present study leads to an overestimation of the cooling effectiveness by about $9 \%$ at zero pressure gradient. The overestimation is similar at shock interaction. Therefore, reasonable conclusions can be drawn from the current study at the present spanwise domain extent.

\section{FLOW CONFIGURATION}

The freestream and injection flow properties match those used in the experiment by Juhany et al. [10]. The freestream Mach number is set to $\mathrm{Ma}_{\infty}=2.44$ and the freestream Reynolds number $\operatorname{Re}_{\infty}=u_{\infty} S / \nu_{\infty}$ based on the slot height $S$, the freestream velocity $u_{\infty}$, and the freestream kinematic viscosity $\nu_{\infty}$ is $\operatorname{Re}_{\infty}=13500$. The freestream boundary-layer thickness at the tip of the lip is $\delta / S=2.2$. The blowing rate $M$, the injection Mach number $\mathrm{Ma}_{i}$, and the total temperature ratio $T_{t i} / T_{t \infty}$ of the cooling flow are listed in Table 1 and are kept constant at all considered cases. Case $\mathrm{I}$ is a zero pressure gradient configuration and is validated in subsection 5.2 by the experiments performed by Juhany et al. [10]. The shock waves caused by a $\beta=5^{\circ}$ flow deflection angle impinge upon the potential-core region in an inviscid flow at a downstream distance $x / S=17$ (case II) and in the boundary-layer region at $x / S=60$ (case III). Additionally, a stronger shock wave is considered impinging at the same downstream distance $x / S=60$ upon the cooling flow (case IV). At the cooling slot, the boundary-layer thickness of the upper and lower laminar supersonic boundary layers are assumed $\delta / S=0.07$. The pressure is set to the freestream pressure $p_{\infty}$.

The duration $\Delta t$ of the simulations which is used for the averaging process is given in Table 1 . The time interval $\Delta t$ is normalized by the timescale $L_{\text {in }} /\left(0.03 u_{\infty}\right)$ where $L_{\text {in }}$ is the interaction length, i. e., the distance between the point of separation and the location where the shock impinges upon the wall assuming inviscid conditions. This time scale is associated with the low frequency motion of the shock [25]. The current time averaging window covers at least one complete cycle of the shock.

Table 1 Flow configuration

\begin{tabular}{ccccccccc}
\hline Case & $\mathrm{Ma}_{i}$ & $T_{t i} / T_{t \infty}$ & $M=\rho_{i} u_{i} /\left(\rho_{\infty} u_{\infty}\right)$ & $\beta$ & $\mathrm{Ma}_{3}$ & $x_{\text {imp }} / S$ & $L_{\text {in }}$ & $\Delta t u_{\infty} \cdot 0.03 / L_{\text {in }}$ \\
\hline I & 1.8 & 0.76 & 0.74 & $0^{\circ}$ & 2.44 & - & - & - \\
II & 1.8 & 0.76 & 0.74 & $5^{\circ}$ & 2.04 & 17 & 7.2 & 1.04 \\
III & 1.8 & 0.76 & 0.74 & $5^{\circ}$ & 2.04 & 60 & 3.2 & 2.58 \\
IV & 1.8 & 0.76 & 0.74 & $8^{\circ}$ & 1.82 & 60 & 5.8 & 1.29 \\
\hline
\end{tabular}




\section{RESULTS}

The results section is divided into two major parts, first, the zero pressure gradient film cooling configuration is validated and the length of the potential-core region is determined. Then, shock-cooling film interactions are investigated in terms of instantaneous and mean flow properties, cooling effectiveness distributions, and turbulence statistics.

\subsection{Zero-Pressure Gradient Configuration (case I)}

The zero-pressure gradient configuration (case I) is validated in section 5.2 by comparing numerically obtained cooling effectiveness distributions with the experiments by Juhany et al. [10].

To determine the length of the potential-core region for the choice of the shock-impingement positions at cases II, III, and IV, streamwise velocity profiles of case I are shown in Fig. $3 a$. At $x / S=0$, the potential-core region is visible by the constant velocity in the slot flow at $-1.16 \leq y / S \leq-0.16$. At increasing downstream distance, this region of constant streamwise velocity shrinks since the laminar slot boundary layer at the wall and the mixing layer, which emanates from the lip, grow and eventually merge. This becomes apparent at the downstream distance $x / S=40$, where the velocity profile already resembles that of an undisturbed fully turbulent boundary layer. The Reynolds shear stress component $\overline{u^{\prime \prime} v^{\prime \prime}} / u_{\infty}^{2}$, which is composed of the fluctuating velocity components in the streamwise and spanwise directions from the Favre-averaged mean, is shown in Fig. $3 b$. The potential-core region is evident by the zero shear stress at $x / S=0$,

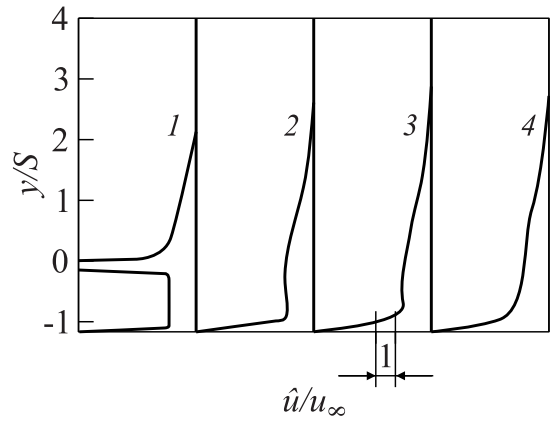

(a)

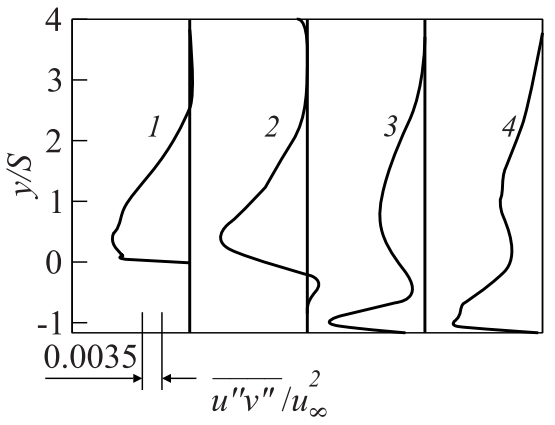

(b)

Figure 3 Streamwise velocity profiles and Reynolds shear stress component $\overline{u^{\prime \prime} v^{\prime \prime}} / u_{\infty}^{2}$ for case I; grid spacing is $\widetilde{u} / u_{\infty}=1$ and $\overline{u^{\prime \prime} v^{\prime \prime}} / u_{\infty}^{2}=0.0035: 1-x / S=0 ; 2-15$; $3-30 ;$ and $4-x / S=40$ 
$-1.16 \leq y / S \leq-0.16$. At $x / S=30$, the laminar slot boundary layer has undergone transition which is indicated by the negative peak shear stress close to the wall. However, a small area with minimal shear stress at $x / S=30, y / S=-0.4$ is still visible, indicating a potential flow. At $x / S=40$, the slot boundary layer and the mixing layer have merged, marking the end of the potential-core region and the beginning of the boundary-layer region.

\subsection{Analysis of Supersonic Film Cooling with Shock Waves}

In the following, cases II, III, and IV are considered, i. e., shock-cooling film interactions are investigated to show the impact on the cooling effectiveness and the turbulence statistics.

\subsubsection{Flow characteristics of supersonic film cooling with shock waves}

Figure $4 a$ shows the skin-friction coefficient distribution vs. streamwise distance of all considered cases to clearly identify regions with separated flow. At the zero-pressure gradient cooling configuration, the skin friction rises quickly at the end of the potential-core region. At the shock-impingement position within the potential-core region at case II, a separation bubble at the length $L_{\text {sep }} / S=6.5$ exists. Downstream of the laminar separation bubble the skin-friction coefficient shows a pronounced peak, which is a clear sign of the transition of the laminar slot boundary layer. At the shock impingement upon the boundary-layer region at case III, a much smaller separation bubble is found with the length of $L_{\mathrm{sep}} / S$ $=0.9$, since the wall-bounded flow is turbulent at this downstream position. The greater shock strength at case IV due to the higher deflection angle $\beta=8^{\circ}$

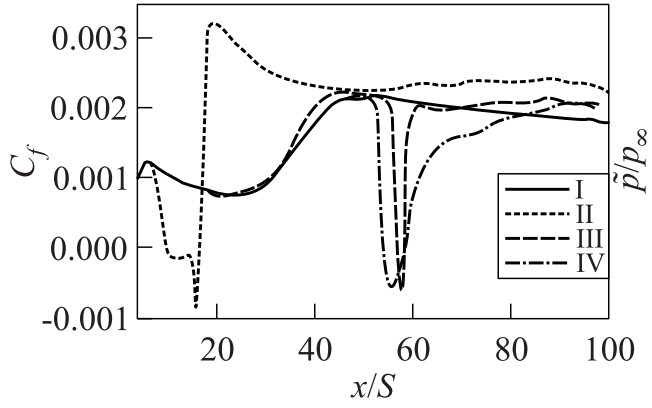

(a)

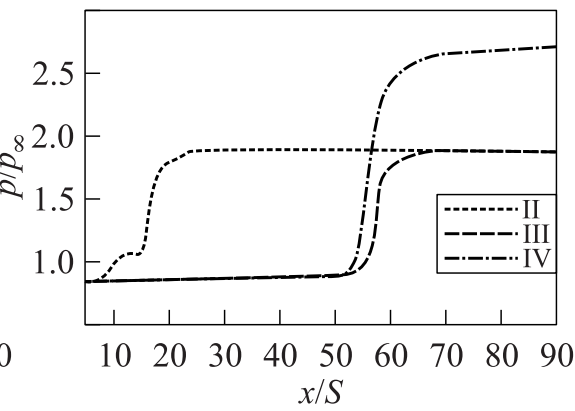

(b)

Figure 4 Skin friction coefficient $(a)$ and wall pressure distribution $(b)$ plotted vs. the streamwise distance from the slot: I to IV refer to cases I to IV 
leads to a slightly larger separation $L_{\mathrm{sep}} / S=3.6$, but the minimum skin-friction coefficient has the same level as at case III.

The wall pressure distributions for cases II, III, and IV are juxtaposed in Fig. $4 b$. The large laminar separation bubble at case II is visible by the plateau in the pressure distribution at $x / S=15$. Such a plateau is not observed at the shock impingement in the boundary-layer region at both shock strengths.

\subsubsection{Instantaneous flow field}

Figure 5 shows the vortical structures visualized by the $Q$ criterion [26] with mapped-on Mach number contours for the shock impinging upon the potentialcore region (case II). Shock waves are indicated by gray contours of the velocity divergence $\left(\partial u_{i} / \partial x_{i}\right) S / u_{\infty}=-0.2$. The incoming turbulent boundary layer above the slot is disturbed by an expansion fan which is followed by a shock wave. In the injected cooling film no vortices are present. The upper border of this region is defined by the mixing and shear layers which emanate from the lip. The incident oblique shock wave penetrates through the shear and mixing layers. Downstream of the reflected shock, the vortices are detected in the cooling flow terminating the potential-core region.

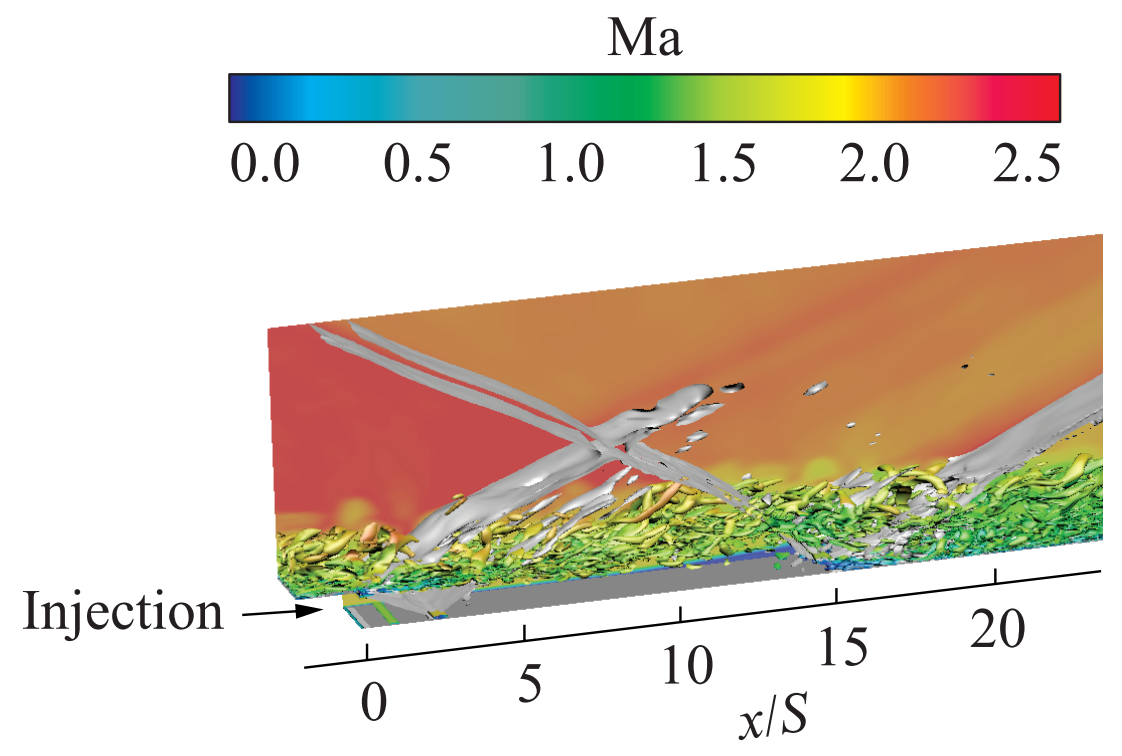

Figure 5 Turbulent structures visualized by the $Q$ criterion with mapped-on Mach number contours; shock- and expansion-fan contours at $\left(\partial u_{i} / \partial x_{i}\right) S / u_{\infty}=-0.2$, case II. 


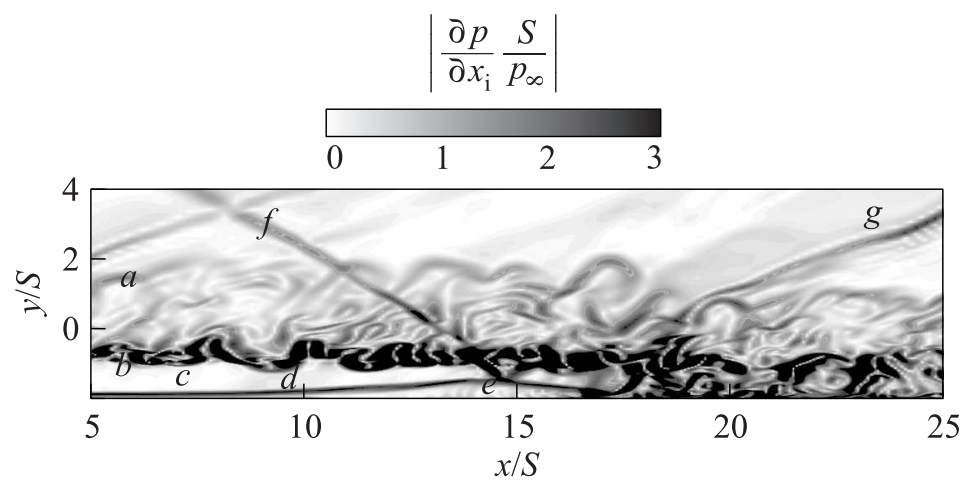

Figure 6 Instantaneous numerical Schlieren image at the centerline of the computational domain at case II: $a$ - shear layer; $b$ - mixing layer; $c$ - potential-core region; $d$ - laminar slot boundary layer; $e$ - laminar separation bubble; $f$ - incident shock wave; and $g$ - reflected shock wave

These flow features at the shock-impingement position within the potentialcore region (case II) are clearly visible in the instantaneous numerical Schlieren image shown in Fig. 6. The potential-core region $(c)$, which is encompassed by the laminar slot boundary layer $(d)$ and the mixing layer $(b)$, extends even downstream of the shock impingement position at this time level to $x / S=14.5$. Further downstream at $x / S=17$, disturbances appear in the potential-core flow which is near the time-averaged reattachment point $x_{R} / S=16.3$ of the separation bubble $(e)$.

\subsubsection{Cooling effectiveness}

The temporal and spanwise averaged cooling effectiveness definition reads

$$
\widetilde{\eta}=\frac{\widetilde{T}_{\mathrm{aw}}-T_{r \infty}}{T_{i \infty}-T_{r \infty}}
$$

where the quantity $\widetilde{T}_{\text {aw }}$ denotes the Favre-averaged adiabatic wall temperature, $T_{r \infty}$ is the recovery temperature of the freestream, and $T_{i \infty}$ is the recovery temperature of the cooling flow. The cooling effectiveness distribution in Fig. $7 a$ for case I shows a good agreement with the experiments of Juhany et al. [10]. An in-depth analysis of this cooling configuration can be found in [23]. Konopka et al. [24] investigated a similar cooling configuration with a lower injection Mach number of $\mathrm{Ma}_{i}=1.2$. Note that Konopka et al. found that the numerical cooling effectiveness is $9 \%$ lower when using a spanwise domain size of $z / D=2.2$ compared to $z / D=4.4$ since some large-scale turbulence is 


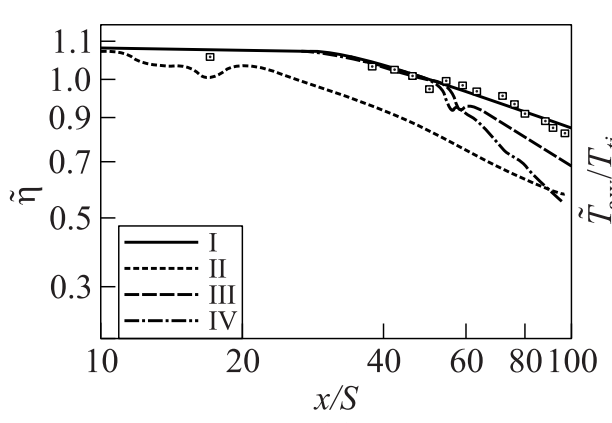

(a)

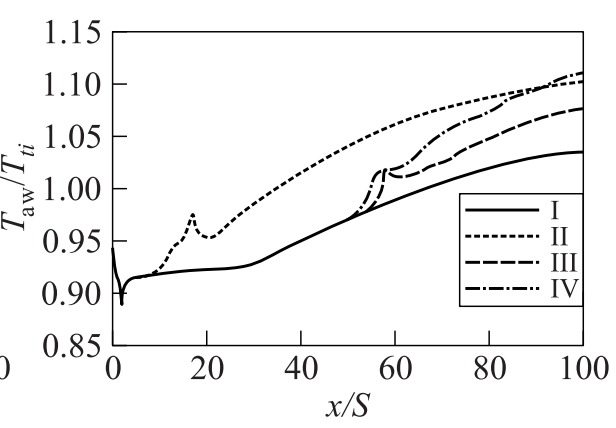

(b)

Figure 7 Cooling effectiveness $(a)$ and wall temperature $(b)$ vs. streamwise distance for cases I-IV. Signs refer to experimental data obtained from [10]

not captured. However, the conclusions drawn in this study are still reasonable since the magnitude of the overestimation is the same for cases I-IV. In Fig. $7 a$, it is evidenced that at the shock impingement upon the potential-core region (case II), the cooling effectiveness is reduced starting from the separation point of the laminar slot boundary layer at $x_{S} / S=9.8$. Downstream of the transition of the laminar slot boundary layer downstream of the separation bubble, the cooling effectiveness decreases further. The turbulent slot boundary layer immediately mixes with the mixing layer emanating from the lip which extends at this downstream position towards the wall. Therefore, it is clear that in case II, besides the reduction of the recovery temperature of the wall by the reduction of the local Mach number, downstream of the shock wave the transition of the laminar slot boundary layer plays an important role. At the more downstream shock impingement position at $x_{\mathrm{imp}} / S=60$ within the boundary-layer region (case III), the streamwise slope of the cooling effectiveness has increased compared to the zero pressure gradient configuration, indicating the increased heat and momentum transfer due to the shock-wave impingement. In case IV, where the flow-deflection angle is increased by $3^{\circ} \mathrm{com}-$ pared to case III, the streamwise slope of the cooling effectiveness decline is even steeper.

The cooling effectiveness values which are slightly above unity at $x / S=10$ are due to the expansion fan and shock wave emanating from the lower tip of the lip which impinge upon the laminar slot boundary layer. This is evidenced by the temperature dip at $x / S=2$ in the wall-temperature distribution in Fig. $7 b$.

Besides the averaged wall temperatures, the frequent changes of the wall temperature have to be considered in the design process of the wall material of a Scramjet engine, as they might lead to thermal fatigue or local hot spots in the engine. Therefore, the cooling effectiveness fluctuations 


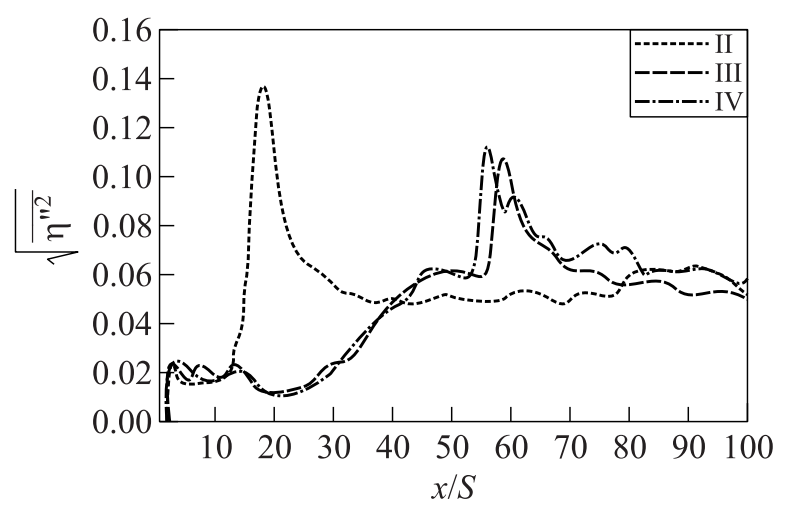

Figure 8 Cooling-effectiveness fluctuation distributions vs. streamwise distance for cases II-IV and instantaneous cooling effectiveness vs. time

$$
\sqrt{\overline{\eta^{\prime \prime 2}}}=\frac{\sqrt{\overline{T_{\mathrm{aw}}^{\prime \prime}}}}{T_{r \infty}-T_{r i}}
$$

are evaluated for the shock-cooling-film interactions in Fig. 8. It is evident that the highest cooling effectiveness fluctuations occur at $x / S=17.82$ at case II, which is downstream of the averaged reattachment point of the separation bubble. At the more downstream shock-impingement position in cases III and IV, the level of cooling effectiveness fluctuations is $16 \%$ lower than in case II and hardly affected by the shock strength, since there is barely any difference between cases III and IV. To investigate the cause for the high cooling effective-

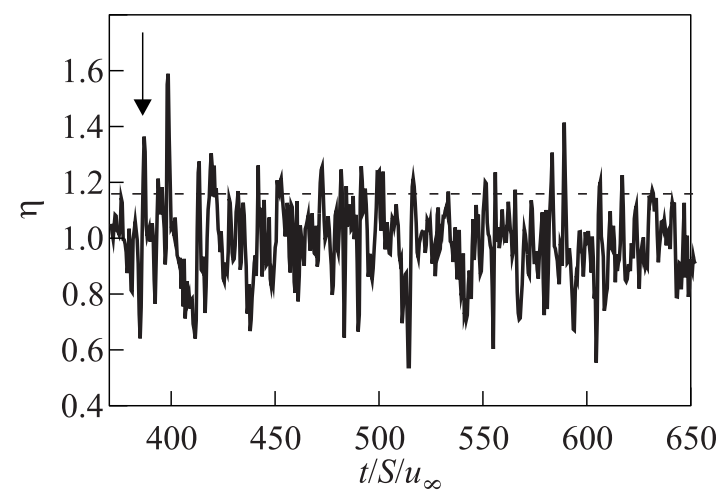

Figure 9 Instantaneous cooling effectiveness at $x / S=17.82, z / S=1.1$ for case II; dashed line corresponds to a cooling effectiveness $\eta=1.1$ 


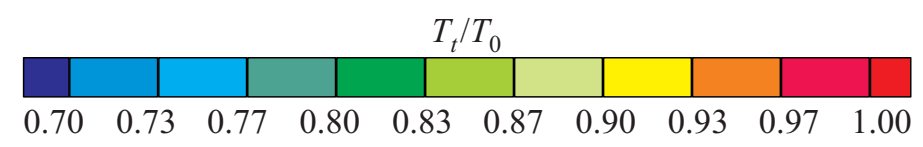

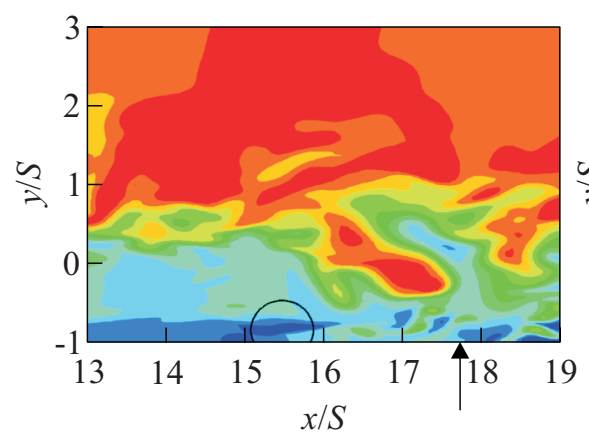

(a)

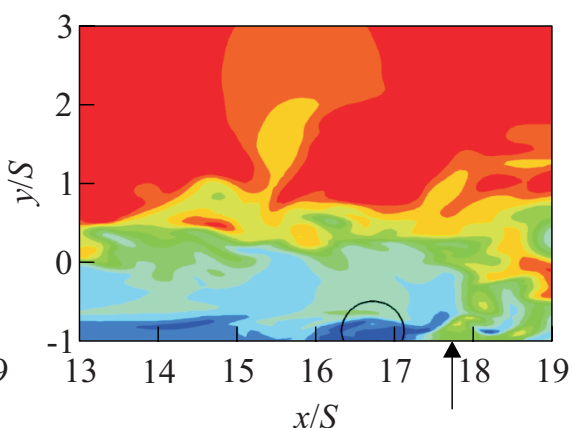

(b)

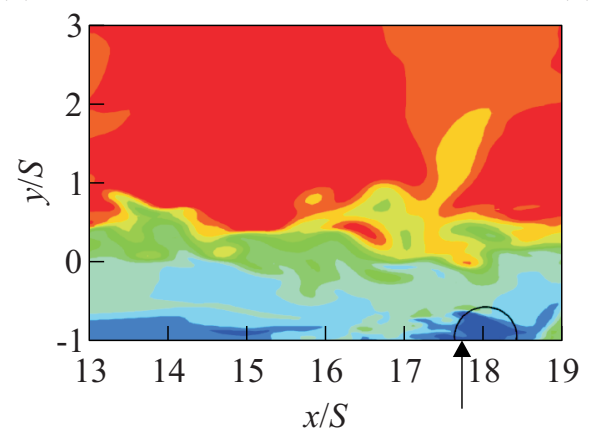

(c)

Figure 10 Instantaneous total temperature contours in the $x, y$-plane at $z / S=1.1$ for case II, the arrows indicate the point of maximum cooling-effectiveness fluctuation and the circles mark the patch of tracked cold fluid: (a) $t / S / u_{\infty}=381 ;(b) 384$; and (c) $t / S / u_{\infty}=386.5$.

ness fluctuation level at the shock impingement upon the potential-core region, the temporal evolution of the cooling effectiveness at the point of maximum cooling effectiveness is shown in Fig. 9. Drastic variations of $\eta$ in the range of $0.5 \leq \eta \leq 1.5$ occur. The sequence of events that leads to the high cooling effectiveness of $\eta=1.3$ at $t_{1} / S / u_{\infty}=386.5$ is shown in Fig. 10. In Fig. 10a, total temperature contours at the center line of the cooling domain are shown. The low energetic fluid in the separation bubble is visible in the bottom left corner. A patch of low energetic fluid is marked by a circle. At a later time $t_{2}$ in Fig. 10b, the patch that has been shed off the separation bubble has moved downstream to $16 \leq x / S \leq 17$. Even later, at $t_{3}$, this patch reaches the po- 


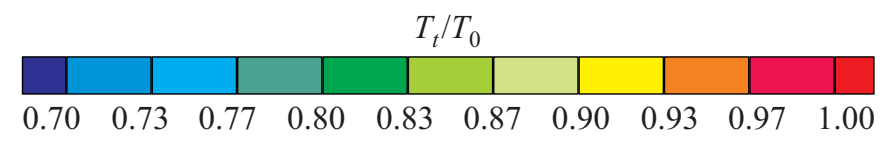

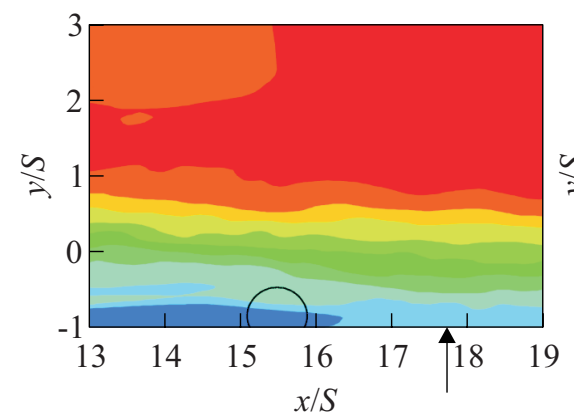

(a)

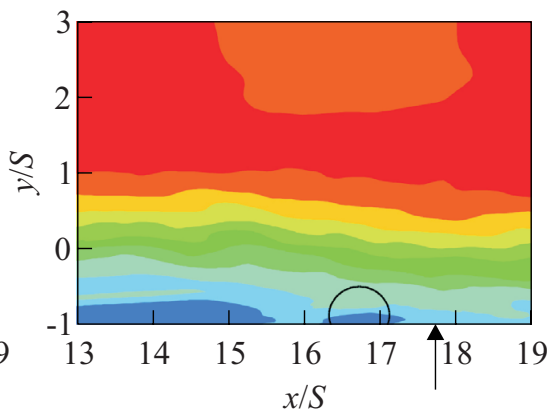

(b)

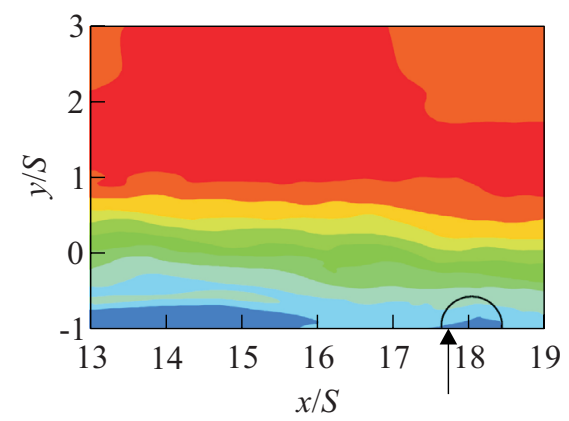

(c)

Figure 11 Conditionally averaged total temperature contours in the $x, y$-plane at $z / S=1.1$ for case II, the arrows indicate the point of maximum cooling-effectiveness fluctuation and the circles mark the regions of low energetic fluid: $(a) \Delta t / S / u_{\infty}=-5.5$; (b) -2.5 ; and $(c) \Delta t / S / u_{\infty}=0.0$.

sition of the maximum cooling-effectiveness fluctuation at $x / S=17.82$ and cools the wall leading to the high cooling effectiveness of 1.3 as observed in Fig. 9.

To further substantiate this finding, conditional averages of the flow field are considered. In Fig. 9, the dashed line corresponds to the averaging threshold, i. e., all snapshots of the flow field with a local cooling effectiveness $\eta \geq 1.1$ are considered. The averaged total temperature contours shown in Fig. 11 consist of 51 snapshots of the flow field. Figure $11 c$ shows the conditionally averaged total temperature contours collected at the time when the condition $\eta \geq 1.1$ holds. It is visible that a region of cold fluid exists at the position of high cooling ef- 
fectiveness fluctuations. The origin of the cold fluid is analyzed by considering the conditionally averaged flow field at $\Delta t / S / u_{\infty}=-2.5$ shown in Fig. $11 b$ before the time when $\eta \geq 1.1$ is satisfied. This time interval corresponds to the time interval between Figs. $10 b$ and $10 c$ in the instantaneous analysis. It is evidenced that the region of cold fluid is now located upstream of the point of maximum cooling effectiveness fluctuations. Considering the conditionally averaged flow field at $\Delta t / S / u_{\infty}=-5.5$ in Fig. $11 a$, it is visible that the region of cold fluid is located upstream compared to the time interval $\Delta t / S / u_{\infty}$ $=-2.5$ and is now a part of the separation bubble. Comparing the time intervals $\Delta t / S / u_{\infty}=-5.5$ and -2.5 , it is seen that the separation bubble has shrunk which means that the region of cold fluid was shed off the separation bubble. Hence, it can be concluded that the same sequence of events shown in the instantaneous analysis in Fig. 10 occurs at all occasions of very high cooling effectiveness.

\subsubsection{Mean flow field}

To analyze the impact of the shock waves on the mixing in the flow field near the wall, the dimensionless fluid temperature

$$
\Theta=\frac{\widetilde{T}_{t}-T_{t \infty}}{T_{t i}-T_{t \infty}}
$$

is evaluated. Its definition is similar to the cooling effectiveness $\widetilde{\eta}$ except that total temperatures are used as reference temperatures. The quantity $\Theta$ reaches a value of 1 in the cooling flow and 0 in the freestream. Figure $12 a$ shows dimensionless fluid temperatures in the slot vicinity for the zero-pressure gra-

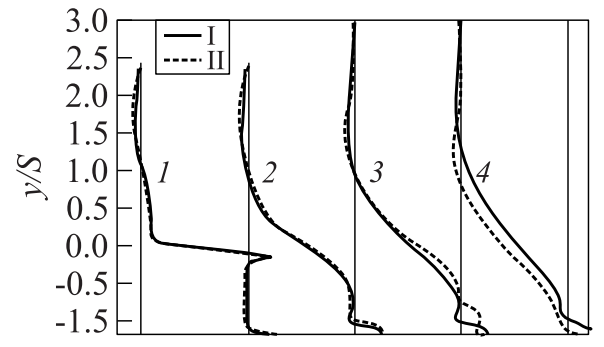

(a)

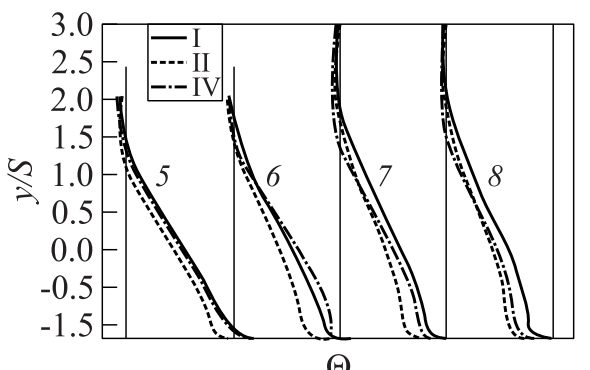

(b)

Figure 12 Dimensionless fluid temperature profiles $\Theta$ for cases I, II, and IV at several downstream positions, grid spacing is $\Delta \Theta=1: 1-x / S=0 ; 2-10 ; 3-15 ; 4-$ $25 ; 5-40 ; 6-60 ; 7-70$; and $8-x / S=80$ 
dient configuration (case I) and the shock-cooling-film interaction within the potential-core region (case II). The spreading of the mixing layer at increasing downstream distance is evident in the profiles, e.g., at $x / S=10$. It is the region where $\Theta$ varies from 1 to 0 at increasing wall-normal distances. Downstream of the shock-wave interaction in case II at $x / S=25$, the dimensionless fluid temperature is lower than at case I indicating increased mixing downstream of the shock compared to the zero-pressure gradient configuration case I. Further downstream, at the stronger shock-cooling-film interaction in the boundary-layer region in case IV, the dimensionless fluid temperature rises first at $x / S=50$ due to the displacement of the separation bubble compared to the case I profile. Further downstream, the dimensionless fluid temperature at case IV is quickly reduced throughout the entire boundary-layer profile and at $x / S=80$ it almost matches the case II profile. At this downstream position, the cooling effectiveness of these two cases is also alike (see Fig. $7 a$ ).

\subsubsection{Turbulence statistics}

Shock waves impinging upon turbulent boundary layers are known [27] to lead to increased turbulence levels downstream of the impingement point. These increased turbulence levels lead to increased mixing and heat and momentum transport of the cooling flow and the freestream.

This is evidenced by the Reynolds shear stress profiles in Fig. 13. In case I, the upper boundary of the potential-core region is located where $\overline{u^{\prime \prime} v^{\prime \prime}} / u_{\infty}^{2}$ begins to deviate from zero at increasing wall-normal distances (Fig. 13a). At increasing streamwise distance from the slot, the upper boundary moves towards the wall. At shock impingement, the profile at $x / S=15$ at case II shows a nega-

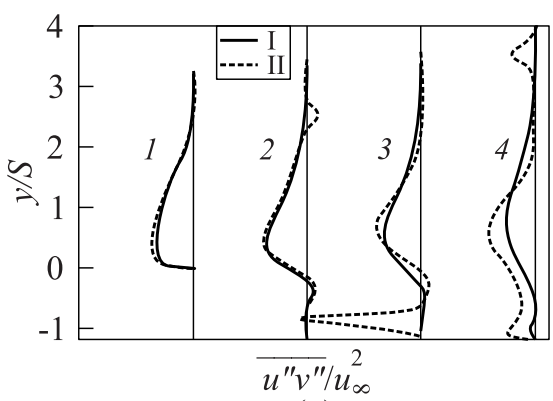

(a)

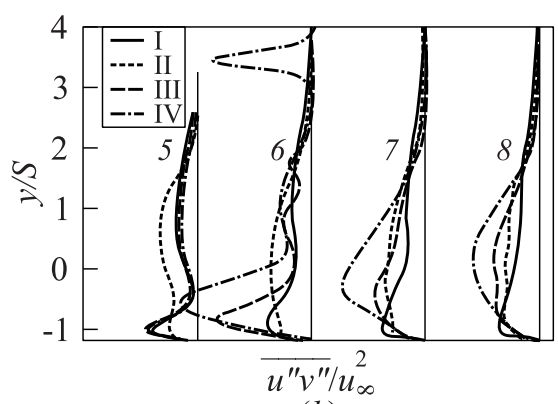

(b)

Figure 13 Reynolds shear stress component for cases I-IV at several downstream positions, grid spacing is $\Delta \overline{u^{\prime \prime} v^{\prime \prime}} / u_{\infty}^{2}=0.0035: 1-x / S=0 ; 2-10 ; 3-15 ; 4-$ $25 ; 5-40 ; 6-60 ; 7-70 ;$ and $8-x / S=80$ 
tive peak at $y / S=-0.9$ nearly at the end of the separation bubble. Further downstream of the shock-impingement position, absolute $\overline{u^{\prime \prime} v^{\prime \prime}} / u_{\infty}^{2}$ levels have risen at $x / S=25,-1.16 \leq y / S \leq 1$. Therefore, it is evident that the shockwave impinging upon the potential-core region has led to the transition of the laminar slot boundary layer and has reduced the length of the potential core region. At shock impingement within the boundary-layer region negative peaks of $\overline{u^{\prime \prime} v^{\prime \prime}} / u_{\infty}^{2}$ are detected at $x / S=60$ close to the wall, which move off the wall further downstream. These higher $\overline{u^{\prime \prime} v^{\prime \prime}} / u_{\infty}^{2}$ values of the cases III and IV than of the cases I and II in the boundary-layer region at $x / S=80$ could explain the steeper streamwise slope in cooling effectiveness observed in Fig. $7 a$.

\section{CONCLUDING REMARKS}

Large-eddy simulations of shock - cooling film interactions have been performed. The shock-waves impinge upon the potential-core (case II) and boundary-layer regions (case III and IV). At the shock-wave impingement position within the potential-core region, the transition of the laminar slot boundary layer occurred downstream of the separation bubble. The increased turbulence levels in the shear- and mixing layer located between the cooling flow and the freestream led to a decrease of the cooling effectiveness compared to the zero-pressure gradient configuration (case I) of $33 \%$. Large cooling effectiveness fluctuations were detected at shock-impingement downstream of the separation bubble since it sheds off large patches of cold fluid. At the shock impingement upon the boundary-layer region at the same shock strength as at the shock impingement upon the potential-core region, a less drastic decrease of cooling effectiveness $(17 \%)$ was observed. However, the streamwise cooling effectiveness gradient showed a higher magnitude (case III) compared to the zero-pressure gradient configuration (case I). At increasing shock strength at the further downstream impingement position (case IV), the cooling effectiveness decreased even more rapidly, i. e., the streamwise cooling effectiveness gradient showed an even higher magnitude.

\section{ACKNOWLEDGMENTS}

The support of this research by the German Research Association (DFG) in the framework of the Research Training Group "Aero-Thermodynamic Design of a Scramjet Propulsion System for Future Space Transportation Systems" 1095/2 and the High Performance Computing Center Stuttgart (HLRS) is gratefully acknowledged. 


\section{REFERENCES}

1. Alzner, E., and V. Zakkay. 1971. Turbulent boundary-layer shock interaction with and without Injection. AIAA J. 9(9):1769-76.

2. Olsen, G. C., R. J. Nowak, M. S. Holden, and N. R. Baker. 1990. Experimental results for film cooling in 2-d supersonic flow including coolant delivery pressure, geometry, and incident shock effects. AIAA Paper No. 90-0605.

3. Holden, M. S., R. J. Nowak, G. C. Olsen, and K. M. Rodriguez. 1990. Experimental studies of shock wave/wall jet interaction in hypersonic flow. AIAA Paper No. 900607.

4. Kanda, T., F. Ono, T. Saito, M. Takahashi, and Y. Wakamatsu. 1996. Experimental studies of supersonic film cooling with shock wave interaction. AIAA J. 34(2):26571 .

5. Kanda, T., and F. Ono. 1997. Experimental studies of supersonic film cooling with shock wave interaction (II). J. Thermophys. Heat Transfer 11(4):590-92.

6. Peng, W., and P.-X. Jiang. 2009. Influence of shock waves on supersonic film cooling. J. Spacecr. Rockets 46(1):67-73.

7. Menter, F. M. 1993. Zonal two equation $k$-omega turbulence models for aerodynamic flows. AIAA Paper No. 93-2906.

8. Juhany, K. A., and M. L. Hunt. 1994. Flowfield measurements in supersonic film cooling including the effect of shock-wave interaction. AIAA J. 32:578-85.

9. Seban, R. A., and L. H. Back. 1962. Velocity and temperature profiles in turbulent boundary layers with tangential injection. J. Heat Transfer 84:45-54.

10. Juhany, K. A., M. L. Hunt, and J. M. Sivo. 1994. Influence of injectant Mach number and temperature on supersonic film cooling. J. Thermophys. Heat Transfer 8(1):5967 .

11. Bowersox, R.D. W., and J. A. Schetz. 1994. Compressible turbulence measurements in a high-speed high-Reynolds-number mixing layer. AIAA J. 32(4):758-64.

12. Aupoix, B., A. Mignosi, S. Viala, F. Bouvier, and R. Gaillard. 1998. Experimental and numerical study of supersonic film cooling. AIAA J. 36:915-23.

13. Sarkar, S. 2000. Numerical simulation of supersonic slot injection into a turbulent supersonic stream. Int. J. Turbo Jet. Eng. 17(3):227-40.

14. Aupoix, B. 2004. Modelling of compressibility effects in mixing layers. J. Turbulence $5(7)$.

15. Liou, M. S., and C. J. Steffen. 1994. A new flux splitting scheme. J. Comput. Phys. 107(1):23-39.

16. Boris, J. P., F. F. Grinstein, E. S. Orana, and R. L. Kolbea. 1992. New insights into large eddy simulation. Fluid Dyn. Res. 10:199-228.

17. Meinke, M., W. Schröder, E. Krause, and Th. Rister. 2002. A comparison of second and sixth-order methods for large-eddy simulations. Comput. Fluids 31:695-718.

18. Rütten, F., W. Schröder, and M. Meinke. 2005. LES of low frequency oscillations of the dean vortices in turbulent pipe bend flows. Phys. Fluids 17(2):035107. 
19. Alkishriwi, N., M. Meinke, and W. Schröder. 2006. A large-eddy simulation method for low mach number flows using preconditioning and multigrid. Comput. Fluids 35(10):1126-36.

20. Renze, P., W. Schröder, and M. Meinke. 2008. Large-eddy simulation of film cooling flows at density gradients. Int. J. Heat Fluid Flow 29(1):18-34.

21. El-Askary, W. A., W. Schröder, and M. Meinke. 2003. LES of compressible wallbounded flows. AIAA Paper No. 2003-3554.

22. Lund, T. S., W. Xiaohua, and K.D. Squires. 1998. Generation of turbulent inflow data for spatially-developing boundary layer simulations. J. Comput. Phys. 140(2):233-58.

23. Konopka, M., M. Meinke, and W. Schröder. 2011. Large-eddy simulation of supersonic film cooling at laminar and turbulent injection. AIAA Paper No. 2011-2250.

24. Konopka, M., M. Meinke, and W. Schröder. 2012. Large-eddy simulation of shockcooling-film interaction. AIAA J. 50(10):2104-14.

25. Piponniau, S., J. P. Dussauge, J. F. Debieve, and P. Dupont. 2009. A simple model for low-frequency unsteadiness in shock-induced separation. J. Fluid Mech. 629:87108.

26. Dubief, Y., and F. Delcayre. 2000. On coherent-vortex identification in turbulence. J. Turbulence 1(11).

27. Priebe, S., M. Wu, and M.P. Martin. 2009. Direct numerical simulation of a reflected-shock-wave/turbulent-boundary-layer interaction. AIAA J. 47:1173-84. 